\title{
Performance evaluation of decision-making agents' in the multi-agent system
}

\author{
Jerzy Korczak, Marcin Hernes, Maciej Bac \\ Wrocław University of Economics ul. Komandorska 118/120, 53-345 Wrocław, Poland \\ e-mail:\{jerzy.korczak, marcin.hernes, maciej.bac\}ue.wroc.pl
}

\begin{abstract}
The article presents the performance analysis issues of buy-sell decisions agents' in a-Trader system. The system allows for supporting of investment decision on FOREX market. The first part of article contains a description of a aTrader system. Next, the algorithms of the selected buy-sell decision agents is presented. In the last part of article the evaluation function of agents' performance is detailed, and the approach to performance analysis is proposed and illustrated.
\end{abstract}

\section{INTRODUCTION}

$\mathrm{S}$ UPPORTING financial decision making process is performed with the use of methods based on mathematics, statistics, economy or artificial intelligence $[2,4,5,10,12,15,17,19]$. The methods are often implemented as the algorithms of the functioning of software agents in multi-agent systems [22]. The paper [1] presents using a multi-agent system in the FOREX market (Foreign Exchange Market). This is one of the biggest financial foreign exchange markets in the world. Currencies are traded against one another in pairs, for instance EUR/USD, USD/PLN. Also a-Trader is the example of system, which enables to support taking investment decisions on the FOREX $[13,14]$. This system used tick data, on the basis of which minute aggregates (M1, M5, M15, M30), hourly aggregates (H1, H4), daily aggregates (D1), weekly aggregates (W1) and monthly aggregates (MN1) are created.

Agents functioning in the system take buy-sell decisions with the use of diversified support methods. There arises the need of constant evaluation of the performance of the agents for the purpose of determining the agents giving advice, in the current market situation, regarding the best decisions. As a consequence, the agents' decisions which are given the highest evaluation may constitute the basis for the performance of the buy-sell transaction by the investor. Return on investment cannot be assumed as the only evaluation criterion because other aspects having influence on the effectiveness of the buy-sell decision taken, such as for instance investment risk [9] or transaction costs should also be taken into consideration.

The purpose of this article is to perform the analysis of the performance of selected agents functioning in the aTrader system with the use of various measures and to elaborate the method of its measurement (evaluation).

In the first part of the article, the a-Trader system is shortly characterized. The algorithms of three selected agents are then presented. In the final part of the article the results of the performance evaluation of these agents is described.

\section{A-TRADER MULTI-AGENT SYSTEM}

The a-Trade platform is of the nature of a multi-agent solution supporting the analysis of high frequency time series, such as e.g. listing of currency pairs on the FOREX market. The basic features of this system include openness, enabling the integration and development of new functionalities of the system and ensuring appropriate communication between particular agents. The system operates in the real time, processing data from the currency market maker live, provided with the use of the MetaTrader or JForex software. After the processing of data provided, the a-Trader system returns the information on making the transaction of the change of the item parameters to the broker (stop loss, take profit). Detailed description of the architecture and its elements as well as information about the agents operating within the framework of the platform may be found in the previous works describing the a-Trader system $[13,14]$. This study describes a sample information flow within the platform. The solution diagram regarding the problem of the transfer of information generated by over one thousand agents with the frequency of up to 100 signals per minute will be presented.

A sample route of the signal delivered by the broker (Data Provider 1) is shown in Fig 1. The information processed goes through all system components:

a. Notification Agent (NA),

b. Historical Data Agent (HDA), 


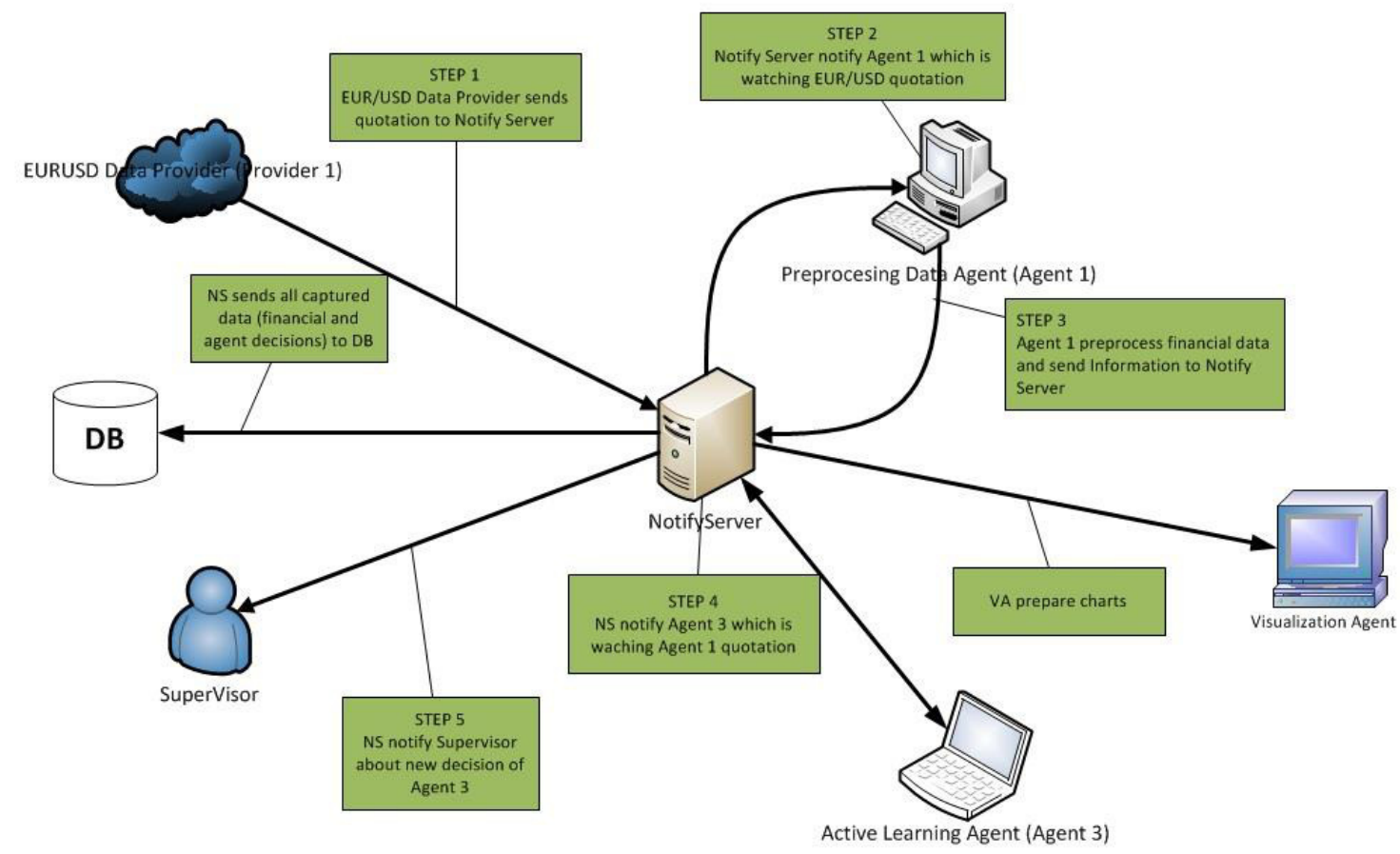

Fig. 1 Signal flow in the A-Trader system. Source: Own work.

c. Cloud of Computing Agents (CCA),

d. Market Communication Agent (MCA),

e. User Communication Agent (UCA),

f. Supervisor (S),

g. System Database (SD),

Information about the change of quotation value goes directly to the Notification Agent (NA) - STEP 1. This agent decides of the further information flow sending it to all agents that listen to a given signal. Independently, the NA sends the signal to the historic data base. In the analysed case the signal is sent to the Data Processing Agent - STEP 2. The Data Processing Agent checks whether the signal is correct and may be subject to analysis by further agents. It sends the verified signal back to the Notification Agent (NA) - STEP 3. NA notifies Agent 3 (Active Learning Agent) about the signal received from Agent 1 - STEP 4. The Active Learning Agent processes information and sends it to the Supervisor through NA - STEP 5. The Supervisor Agent, on the basis of the decision of Active Learning Agent and the decisions of other agents, takes the final decision concerning the transaction. It sends it back to NA which saves it in the data base and sends to the market through the Data Provider Agent 1.

The Notification Agent (NA) ensures efficient communication inside the system. It is the intermediary agent in sending signals between agents as per declared indications (see Fig. 1). Each agent, the status of which changes, notifies its notification agent. The notification agents forwards the information about the change of the status of a given agent to all agents which are recorded in the notification register as clients/observers of its signals. The notification takes place by calling an appropriate web method (SOAP) at all agents from the list of the ones listening to the indicated signals. Then it records the information about the change of the status of the notification agent in the data base. The functionality of the notification agent elaborated this way makes the system flexible and scalable, gives the possibility of simple adding and removing agents and ensures making the system independent of the agent's location.

In order to be able to efficiently manage communication, the Notification Agent operates in a multi-threaded way. Information concerning the message flow, so which agent awaits signals from which agent, is read during the Notification Agent initialization to the Routing Table. Paralelly threads sending information to particular agents are created (Sending Threads Table). The sending threads are responsible for sending information to listening agents. After the creation they check whether the agent to which they are supposed to deliver the information is active and whether it is listening at a given addresses and is ready for processing data. Fig 2 shows the exemplary data flow inside the Notification Agent. The NA listens at the indicated port XXXX. After receiving information from Agent 4 it finds in the Routing Table which agents listen to the signals of Agent 


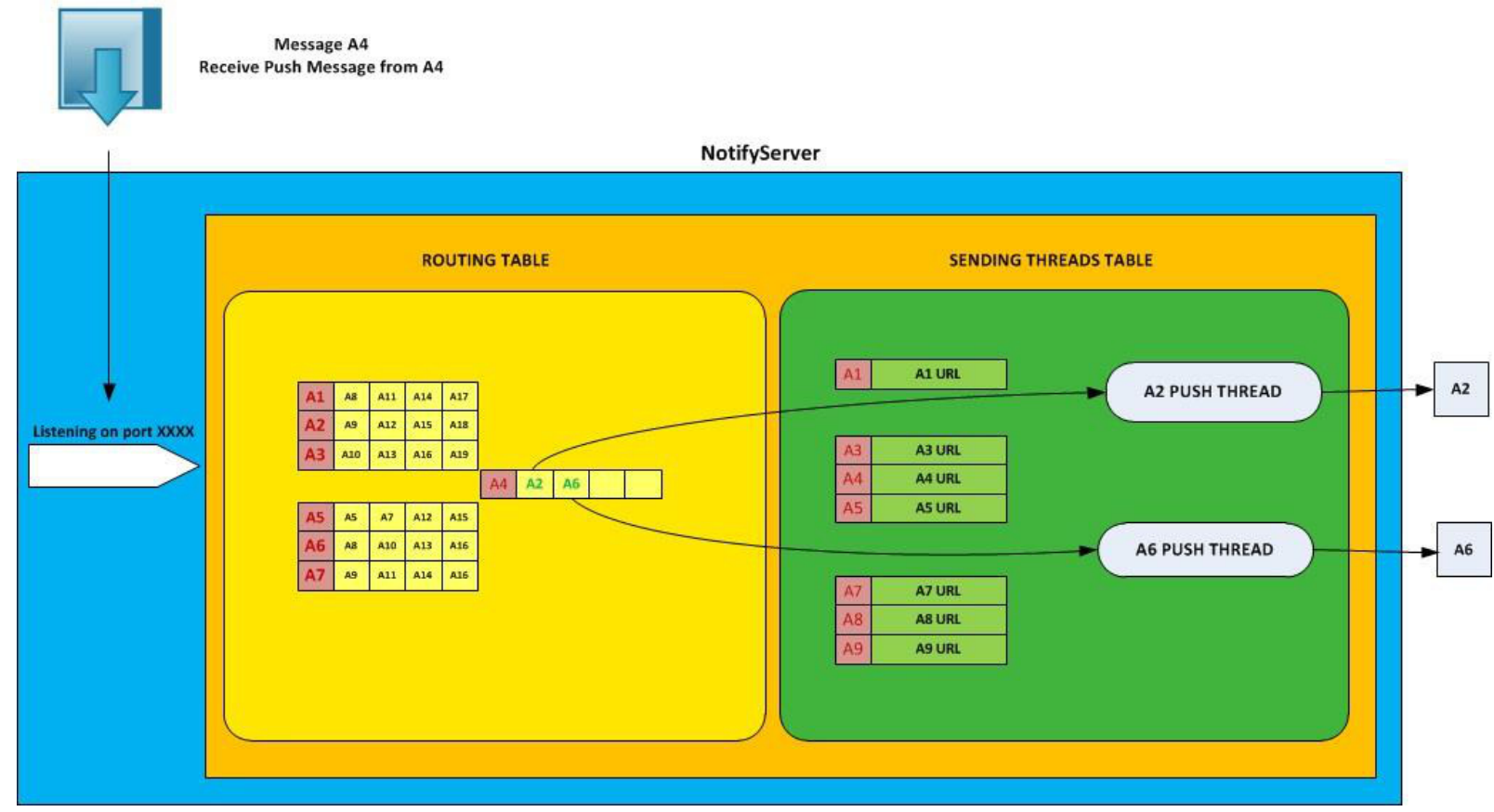

Fig. 2 Data flow inside the Notification Agent. Source: Own work.

4. In the analysed case these are Agent 2 and 6. Then, the Notification Agent finds the threads sending to Agents 2 and 6 and it forwards the information received from Agent 4 to them, sending threads forward the received information from agents with which they communicate. In case when a problem with communication with agents occurs, the sending thread goes into inactive mode not to load the system. The information about the agent's inactivity is recorded in the event $\log$. The inactive sending thread checks if the communication with the agent to which the information is sent can be established. In the case if the inactive agent starts working again, the communication is resumed.

The presented solution enables complete scalability of the platform. In case of excessive loading of the Notification Agent, another instance is activated. The Agents are notified about being assigned a new Notification Agent and they send their signals to it. One NA instance may receive signals from the group of agents, and the received signal must be sent to all agents awaiting given information.

The information received by the Notification Agent may be divided into three groups. The information division is presented in fig 3. The first one is standard information containing the signal generated by any agent working within the framework of the a-Trader system. The second group of information is the control commands. The third group is the warnings and errors sent by agents. Information flow from the first group was already described. Control information is used for managing the Notification Agent. With the use of this information the agents may demand sending information from other agents or demand that selected agents cease sending information. With the use of the control information an agent may resume its activity, the Notification Agent will then start sending signals to it. In case when an agent is being switched off, it should send information to the NA that it wants to stop listening. The sending thread will go into an inactive mode. The third group of information is used for forwarding the information about errors and warnings of particular agents to the central data base. It is sent to NA in the event of the occurrence of exceptional situations in the agent's operation. They include, e.g. too heavy load of an agent, receiving information which it should not receive and other exceptional situations.

The presented flow of the signal inside the a-Trader system allows for better understanding of processes occurring in the system. The agents described in the next part of the article operate in accordance with the presented convention. They accept other agents' signals generated in real time, process them and take financial decisions. The presented technological grounds of the system operation guarantee its scalability. The control messages, messages about errors and warnings increase the system reliability. This enables to process, almost in real time, thousands of signals generated by agents.

From the point of view of the user (investor) the most important components of the a-Trader system are the agents setting the buy-sell decisions (belonging to the cloud of computing agents) and the Supervisor agent. The functioning of these agents enables the investor to make transactions at 


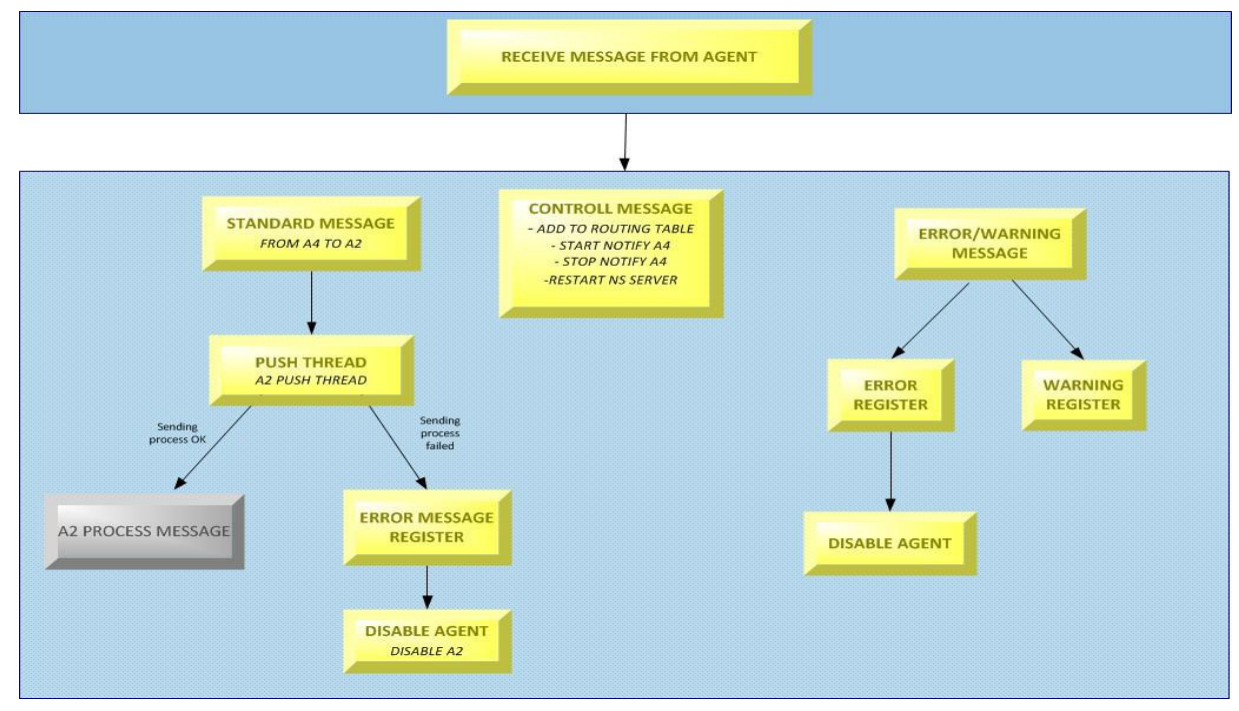

Fig. 3 Types of information received by the Notification Agent. Source: Own work.

the FOREX market, in accordance with signals generated by them. The characteristics of selected agents setting purchasesale transactions will be presented.

\section{DESCRIPTION OF THE BUY-SELL DECISION AGENTS}

Approximately 1000 agents function in the a-Trader system, including approx. 800 agents processing data concerning quotations at the FOREX market (for instance they calculate trend indicators, oscillators) and 200 agents (functioning in all time periods) setting the buy-sell decision. For the needs of this article, in order to perform the efficiency analysis, three agents were selected: TrendLinearReg, MultiTrendSignal and Consensus. These are agents taking decisions on the basis of more complex algorithms than the algorithms of typical technical analysis indicators. The specific nature of the functioning of selected agents will be presented in the further part of the article.

\section{A. . The TrendLinearReg agent}

The agent functions on the basis of the assumption that the trend of a certain number $M$ of quotations is approximated with the straight line with the equation: $y=a x$ $+b$. The straight line inclination depends on the value of the "a" parameter or the tangent value of the inclination angle with the use of linear regression [11, 21]. The agent generates the purchase signal when the coefficient value changes from positive to negative and the sales signal is generated when the coefficient changes value from negative to positive. The change of the agent's decision is made with the use of hysteresis, the level of which is defined by means of the coefficient $\Delta$, the value of which should be higher than transaction costs.

The TrendLinearReg agent functioning algorithm is as follows:
Data: The vector of quotation value of the currency pair $w=<w_{1}, w_{2}$, .... $w_{M}>$ consisting of $M$ quotations and the previous value the $a$ coefficient marked as preva.

Result: The $D$ decision (value 1 denotes „buy” decision, value -1 denotes "sell" decision, value 0 denote $s$, leave unchanged") with respect to $w$ and preva value.

\section{BEGIN}

1: Let $i:=1 ;$ sumy $:=0$; sum $x:=0.0$; sum $x y:=0$; sum $x 2=0$. /I where: sumy means the sum of the value of $M$ quotations, sumx means the sum of the quotation number in the vector, sumxy means the sum of the products of the quotation value and quotation number in the vector, and sumx2 means the sum of the squares of quotation numbers in the vector.

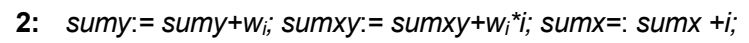
sum $\times 2:=\operatorname{sum} \times 2+i * i ; i:=i+1$;

3: If $i<M$ then go to 2 . If $i \geq N$ then go to: 4 .

4: $c:=\operatorname{sum} x 2^{*} M$-sum $x^{*}$ sumx. If $c=0$ then $c:=0,1$.

5: $a:=\left(\right.$ sum $x y^{*} M-$ sum $x^{*}$ sumy $) / c$;

6: If $(a(+/-\Delta)=$ preva $=0)$ or $((a+\Delta)<0$ and preva $<0)$ or $((a-\Delta)>0$ and preva>0) then $D:=0$;

If $((a+\Delta)<0$ and preva>0) then $D:=1$; If $((a-\Delta)>0$ and preva $<0)$ then $D:=-1$;

7: preva:=a;

END

The complexity of the algorithm, significant due to minimizing of the agent reaction time, amounts to $\mathrm{O}(\mathrm{M})$, where $\mathrm{M}$ means the number of quotations.

\section{B. . The MultiTrendSignal agent}

Agent MultiTrendSignal generates the purchase-sales decision on the basis of the decision of agents functioning with the use of most often used technical analysis ratios [3, 
16]. The decisions of the following nine base agents are analysed:

- Average Directional Index (ADX),

- Relative Strength Index (RSI),

- Rate of Change (ROC),

- Commodity Channel Index (CCI),

- Moving Average of Oscillator (OsMA),

- Moving Average Convergence Divergence (MACD),

- Stop and Reverse (SAR),

- Williams \% R,

- Moving Average (MA).

The agent considers four time periods (M1, M5, M15, M30) in such a way that the buy/sell decision in case of the period M30 is taken when the same decision is taken by most base agents also within the periods M1, M5, M15, M30.

The structure of the investment decision was defined in the study [7]. This decision is taken on the basis of financial instrument quotation such as, e.g. currency pairs EUR/USD, USD/GBP and it is defined as follows:

\section{Definition 1.}

Decision D about finite set of financial instruments $\mathrm{E}=\left\{\mathrm{e}_{1}, \mathrm{e}_{2}, \ldots, \mathrm{e}_{\mathrm{N}}\right\}$ is defined as a set

$$
\mathrm{D}=\left\langle\left\{\mathrm{EW}^{+}\right\},\left\{\mathrm{EW}^{ \pm}\right\},\left\{\mathrm{EW}^{-}\right\}, \mathrm{Z}, \mathrm{SP}, \mathrm{DT}\right\rangle,
$$

where:

1) $\mathrm{EW}^{+}=\left\langle\mathrm{e}_{\mathrm{o}}, \mathrm{pe}_{\mathrm{o}}\right\rangle,\left\langle\mathrm{e}_{\mathrm{q}}, \mathrm{pe}_{\mathrm{q}}\right\rangle, \ldots,\left\langle\mathrm{e}_{\mathrm{p}}, \mathrm{pe}_{\mathrm{p}}\right\rangle$ - a positive set; in other words, it is a set of financial instruments about which the agent knows the decisions to buy, and the volume of this buying.

2) $\mathrm{EW}^{ \pm}=\left\langle\mathrm{e}_{\mathrm{r}}, \mathrm{pe}_{\mathrm{r}}\right\rangle,\left\langle\mathrm{e}_{\mathrm{s}}, \mathrm{pe}_{\mathrm{s}}\right\rangle, \ldots,\left\langle\mathrm{e}_{\mathrm{t}}, \mathrm{pe}_{\mathrm{t}}\right\rangle$ - a neutral set, in other words, it is a set of financial instruments, about which the agent does not know that buy or sell. If these instruments are held by an investor, that they should not be sold, or if they are not in possession of the investor, should not be bought by them.

3) $\mathrm{EW}^{-}=\left\langle\mathrm{e}_{\mathrm{u}}, \mathrm{pe}_{\mathrm{u}}\right\rangle,\left\langle\mathrm{e}_{\mathrm{v}}, \mathrm{pe}_{\mathrm{v}}\right\rangle, \ldots,\left\langle\mathrm{e}_{\mathrm{w}}, \mathrm{pe}_{\mathrm{w}}\right\rangle$ - a negative set; in other words it is a set of financial instruments of which the agent knows that these elements should sell. Couple $\left\langle\mathrm{e}_{\mathrm{x}}, \mathrm{pe}_{\mathrm{x}}\right\rangle$, where: $\mathrm{e}_{\mathrm{x}} \in \mathrm{E}$ and $\mathrm{pe}_{\mathrm{x}} \in[0,1]$, denote financial instrument and this instrument's participation in set $\mathrm{EW}^{+}, \mathrm{EW}^{ \pm}, \mathrm{EW}^{-}$.

Financial instrument $\mathrm{e}_{\mathrm{x}} \in \mathrm{EW}^{+}$is denoted as: $\mathrm{e}_{\mathrm{x}}^{+}$.

Financial instrument $e_{x} \in E W^{ \pm}$is denoted as: $e_{x}^{ \pm}$.

Financial instrument $\mathrm{e}_{\mathrm{x}} \in \mathrm{EW}^{-}$is denoted as: $\mathrm{e}_{\mathrm{x}}^{-}$.

4) $\mathrm{Z} \in[0,1]$ - predicted rate of return.

5) $\mathrm{SP} \in[0,1]$ - degree of certainty of rate $Z$. It can be calculated on the basis of the level of risk related with the decision.
6) DT - date of decision.

The set of agents' decisions, on the basis of which the MultiTrendSignal agent sets decisions is called a profile. .

The agent functioning algorithm is as follows;

Data: Profiles $A M 1=\left\{A M 1^{(1)}, A M 1^{(2)}, \ldots . A M 1^{(9)}\right\}$

$A M 5=\left\{A M 5^{(1)}, A M 5^{(2)}, \ldots A M 5^{(9)}\right\}$

$A M 15=\left\{A M 15^{(1)}, A M 15^{(2)}, \ldots . A M 15^{(9)}\right\}$

$A M 30=\left\{A M 30^{(1)}, A M 30^{(2)}, \ldots . A M 30^{(9)}\right\}$

consist of 9 agents' decisions.

Result: Decision $D E C=\left\langle D E C_{+}, D E C_{ \pm}, D E C_{-}, D E C_{Z}, D E C_{S P}, D E C_{D T}\right\rangle$ according the profiles.

BEGIN

1: Let $D E C_{+}=D E C_{ \pm}=D E C_{-}=\varnothing, D E C_{Z}=D E C_{S P}=D E C_{D T}=0$.

2: $j:=1$.

3: $i:=+$.

4: If $\left(t M 1_{i}(j)>4\right)$ and $\left(t M 5_{i}(j)>4\right)$ and $\left(t M 15_{j}(j)>4\right)$ and $\left(t M 30_{i}(j)>4\right)$

then $D E C_{i}=D E C_{i} \cup\left\{e_{j}\right\}$. Go to:6. // $t M x X_{i}(j)$ - the number of occurrences of the financial instrument in a positive, neutral or negative set in a given time period.

5: If $i=+$ then $i:= \pm$. Ifi $i= \pm$ then $i:=-$. if $i=-$ then go to: 6 .

Go to: 4

6: If $j<N$ then $j:=j+1$ go to:3.

If $j \geq N$ then go to: 7 .

7: $i:=Z$.

8: Determine $\operatorname{pr}(i)$. //ascending order

9: $k_{i}^{1}=(9+1) / 2, k_{i}^{2}=(9+2) / 2$.

10: $k_{i}^{1} \leq D E C_{i} \leq k_{i}^{2}$

11: If $i=Z$ then $i:=S P$. If $i:=S P$ then $i:=D T$. Ifi $i:=D T$ then END.

Go to: 8.

END.

The algorithm complexity amounts to $\mathrm{O}(18 \mathrm{~N})$, where $\mathrm{N}$ means the number of currency pairs.

\section{The Consensus agent}

In the a-Trader system the agents take buy-sell decisions independently of one another. Thus a conflict situation may occur, in which (at a given moment) these decisions are mutually contradictory (for instance some agents suggest the purchase decision and other agents - the sale decision). In order to solve this conflict a few strategies were implemented into the system: the strategy of dominating decisions, the strategy based on moving average, the consensus strategy, the evolution strategy. The Consensus method $[8,18,20]$ is described in the article.

The Consensus agent (characterized in detail in the work of [14] determines the decisions on the basis of the set of decisions generated by other agents functioning in the system.

The agent functioning algorithm is as follows:

Data: The profile $A=\left\{A^{(1)}, A^{(2)}, \ldots . A^{(M)}\right\}$ consist of $M$ agents' decisions. $/ / A^{(1)}, A^{(2)}, \ldots . A^{(M)}$ - decisions of particular agents

Result: Consensus

$C O N=\left\langle C O N_{+}, C O N_{ \pm}, C O N_{-}, C O N_{Z}, C O N_{S P}, C O N_{D T}\right\rangle$ according $A$. 


\section{BEGIN}

1: Let $C O N_{+}=C O N_{ \pm}=C O N_{-}=\varnothing, C O N_{Z}=C O N_{S P}=C O N_{D T}=0$.

2: $j:=1$.

3: $i:=+$.

4: If $t_{i}(j)>M$ then $\operatorname{CON}_{i}=\operatorname{CON}_{i} \cup\left\{e_{j}\right\}$. Go to: 6 .

$/ / t_{i}(j)$ - the number of occurrences of the financial instrument in the positive, neutral or negative set

5: If $i=+$ then $i:= \pm$

If $i= \pm$ then $i:=$

If $i=-$, then Go to: 6

Go to: 4 .

6: If $j<N$ then $j:=j+1$ Go to:3

If $j \geq N$ then Go to: 7 .

7: $i:=Z$.

8: Determine $p r(i) . / /$ ascending order

9: $k_{i}^{1}=(M+1) / 2, k_{i}^{2}=(M+2) / 2$.

10: $k_{i}^{1} \leq \mathrm{CON}_{i} \leq k_{i}^{2}$.

11: If $i=Z$ then $i:=S P$.

If $i:=S P$ then $i:=D T$.

If $i:=D T$ then END.

Go to: 8.

END.

The complexity of the algorithm amounts to $\mathrm{O}(3 \mathrm{NM})$, where $\mathrm{N}$ means the number of currency pairs and $\mathrm{M}$ means the number of agents belonging to the profile (in the research experiment conducted in the next part of the article, $M=25$, $\mathrm{N}=1$ ).

Computational complexities of the algorithms of the agents' functioning have impact on the performance of the whole a-Trader system. Taking into consideration the fact that the system is processing tick signals and a large number of agents function in it (approx. 1000), short time of computation made by particular agents is very significant.

In general, agents functioning in the system for the purpose of determining the decisions use the methods of technical analysis, fundamental analysis, neural networks, evolution algorithms, behavioural models.

The Supervisor agent also functions in the system and its major purpose is to maximize the rate of return and reduce the investment risk. The Supervisor's task is to coordinate the functioning of agents setting the buy-sell decisions and presenting the final decision to the investor. This agent uses various strategies, analyses them and evaluates the agents' performance.

A case study relating to the method of the measurement of the performance of selected agents taking buy-sell decisions is presented further in the article.

\section{THE AGENT PERFORMANCE EVALUATION METHOD - CASE STUDY}

The agents performance analysis is performed for data within the M5 range of quotations from the FOREX market. For the purpose of this analysis, a test was performed in which the following assumptions were made:

1. EUR/USD pairs, out of four randomly selected pairs of the following periods, were used:

- 31-03-2014, 0:00 am to 31-03-2014, 23:59 pm,

- 03-04-2014, 0:00 am to 03-03-2014, 23:59 pm,

- 04-04-2014, 0:00 am to 04-04-2014, 23:59 pm,

- 05-04-2014, 0:00 am to 05-04-2014, 17:00 pm,

2. At the verification the decisions (signals buy-value 1 , sell-value - 1 , leave unchanged - value 0 ) generated by the agents TrendLinearReg, MultiTrendSignal are used (the example is presented in the Fig 4, where the green arrow means the decision "buy", the red one "sell"), and Consensus.

3. It was assumed that the initial capital held by the investor amounts to USD 1000 and the difference between this amount and the amount which the investor will have after the last sales transaction in a given period is considered the rate of return. The rate of return is expressed in nominal units (USD).

4. The transaction costs are directly proportional to the number of transactions.

5. The capital management - it was assumed that in each

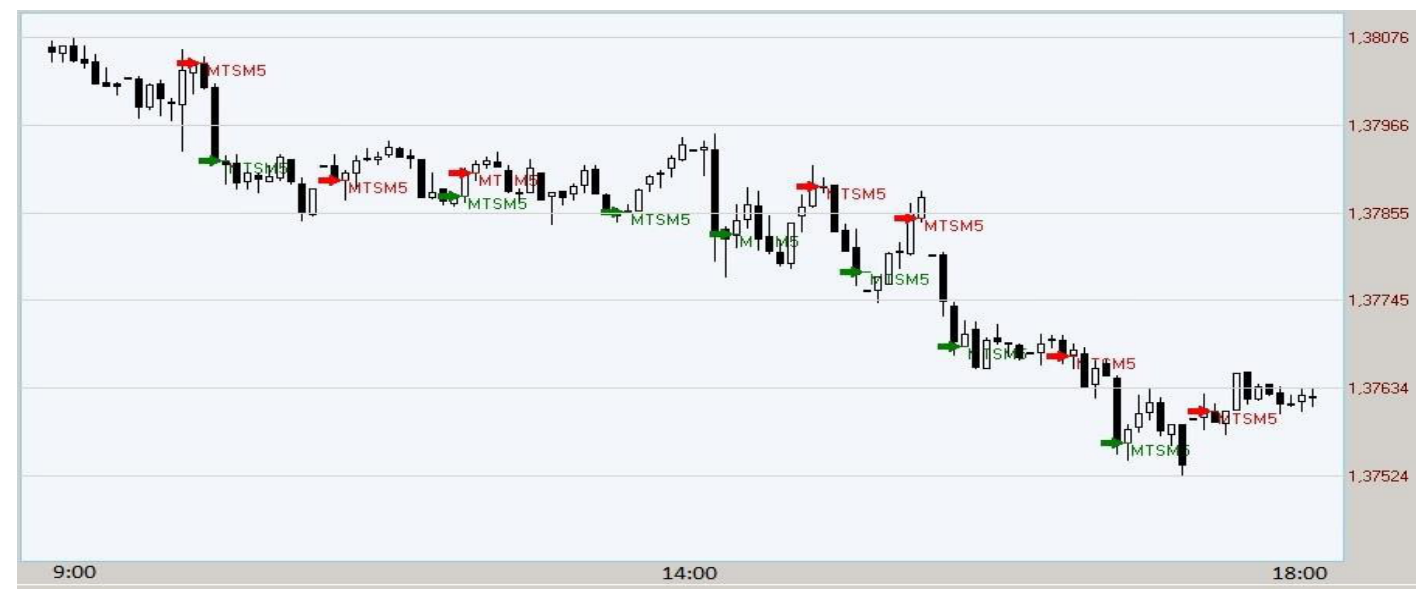

Fig. 4 The MultiTrendSignal agent decisions.

Source: Own work. 
transaction the investor engages $100 \%$ of the capital held. The capital management strategy may be determined by the user. The investor every time invests 1000 at the leverage 10:1 and invests all the capital held.

6. The performance analysis was performed with the use of the following measures (ratios):

- rate of return (ratio $x_{1}$ ),

- the number of transaction,

- gross profit (ratio $\mathrm{x}_{2}$ ),

- gross loss (ratio $x_{3}$ ),

- total profit (ratio $\mathrm{x}_{4}$ ),

- the number of profitable transactions ( $\operatorname{ratio} \mathrm{x}_{5}$ ),

- the number of profitable transactions in a row (ratio $\mathrm{x}_{6}$ ),

- the number of unprofitable transactions in a row $\left(\right.$ ratio $\left.\mathrm{x}_{7}\right)$,

- Sharpe ratio (ratio $\mathrm{x}_{8}$ )

$$
S=\frac{E(r)-E(f)}{|O(r)|} \cdot 100 \%
$$

where:

$\mathrm{E}(\mathrm{r})$ - arithmetic average of the rate of return,

$\mathrm{E}(\mathrm{f})$ - arithmetic average of the risk-free rate of return,

$\mathrm{O}(\mathrm{r})$ - standard deviation of rates of return.

- the average coefficient of variation (ratio $\mathrm{x}_{9}$ )is the ratio of the average deviation of the arithmetic average multiplied by $100 \%$ and is expressed:

$$
\mathrm{V}=\frac{\mathrm{s}}{\mathrm{E}(\mathrm{r}) \mid} \cdot 100 \% \text {. }
$$

where:

$\mathrm{V}$ - average coefficient of variation,

$\mathrm{s}$ - average deviation of the rates of return,

$\mathrm{E}(\mathrm{r})$ - arithmetic average of the rates of return.

- Value at Risk (ratio $x_{10}$ ) - the measure known as value exposed to the risk - that is the maximum loss of the market value of the financial instrument possible to bear in a specific timeframe and at a given confidence level [3].

$$
\mathrm{VaR}=\mathrm{P} * \mathrm{O} * \mathrm{k}
$$

where:

$\mathrm{P}$ - the initial capital,

$\mathrm{O}$ - volatility - standard deviation of rates of return during the period,

$\mathrm{k}$-the inverse of the standard normal cumulative distribution (assumed confidence level 95\%, the value of $\mathrm{k}$ is 1,65 ),

- the average rate of return per transaction (ratio $\mathrm{x}_{11}$ ), counted as the quotient of the rate of return and the number of transactions.
7. For the purpose of the comparison of the agents' performance, the following evaluation function was elaborated:

$\mathrm{y}=\left(\mathrm{a}_{1} \mathrm{x}_{1}+\mathrm{a}_{2} \mathrm{x}_{2}+\mathrm{a}_{3}\left(1-\mathrm{x}_{3}\right)+\mathrm{a}_{4} \mathrm{x}_{4}+\mathrm{a}_{5} \mathrm{x}_{5}+\mathrm{a}_{6} \mathrm{x}_{6}+\ldots\right.$

$\left.+a_{7}\left(1-x_{7}\right)+a_{8} x_{8}+a_{9}\left(1-x_{9}\right)+a_{10}\left(1-x_{10}\right)+a_{11} x_{11}\right)$

where $x_{i}$ denote the normalized values of ratios mentioned in item 6 from $\mathrm{x}_{1}$ to $\mathrm{x}_{11}$. It was adopted in the test that coefficients $a_{1}$ to $a_{11}=1 / 11$.

It should be mentioned that these coefficients may be modified with the use of, for instance, an evolution method or determined by the user (investor) in accordance with his/her preference (for instance the user may determine whether he/she is interested in the higher rate of return with simultaneous higher risk level or lower risk level but simultaneously agrees to a lower rate of return).

The function is given the values from the range [0..1], and the agent's efficiency is directly proportional to the function value.

8. The results obtained by the tested agents were compared with the results of the Buy-and-Hold strategy and the strategies using EMA..

The agent efficiency tests were performed in the following manner:

1. On the basis of data from the first period each agent defined when to buy and when to sell EUR/USD currency.

2. In the next step, on the basis of the results of particular agents and Buy-and-Hold and EMA, for each purchase-sale operation the value of capital held and the rate of return in USD were determined.

3. At the final stage the value of performance ratios was calculated with respect to the rates of return resulting from all decisions generated by the analysed agents and the Buy-and-Hold as well as EMA strategies (not only from the final rates of return but from all rates of return calculated after each sales decision). The evaluation functions were also calculated.

4. Then the steps from 1 to 3 were repeated with the use of the data from the successive periods.

Table 1 presents the results obtained in the particular periods.

Generalizing the agent efficiency analysis results, it may be noticed that in the periods in question their decisions generated both profit and loss. Thus, in the efficiency analysis not only the rate of return should be taken into consideration but also other ratios, including the level of risk involved in the investment, which is enabled by the evaluation function elaborated in the article.

In fig 5 the diagram of the value of ratios and evaluation function of particular agents (and the $\mathrm{B} \& \mathrm{H}$ method as well as EMA) in the periods in question are presented. To illustrate relationships between ratios and agents the parallel 


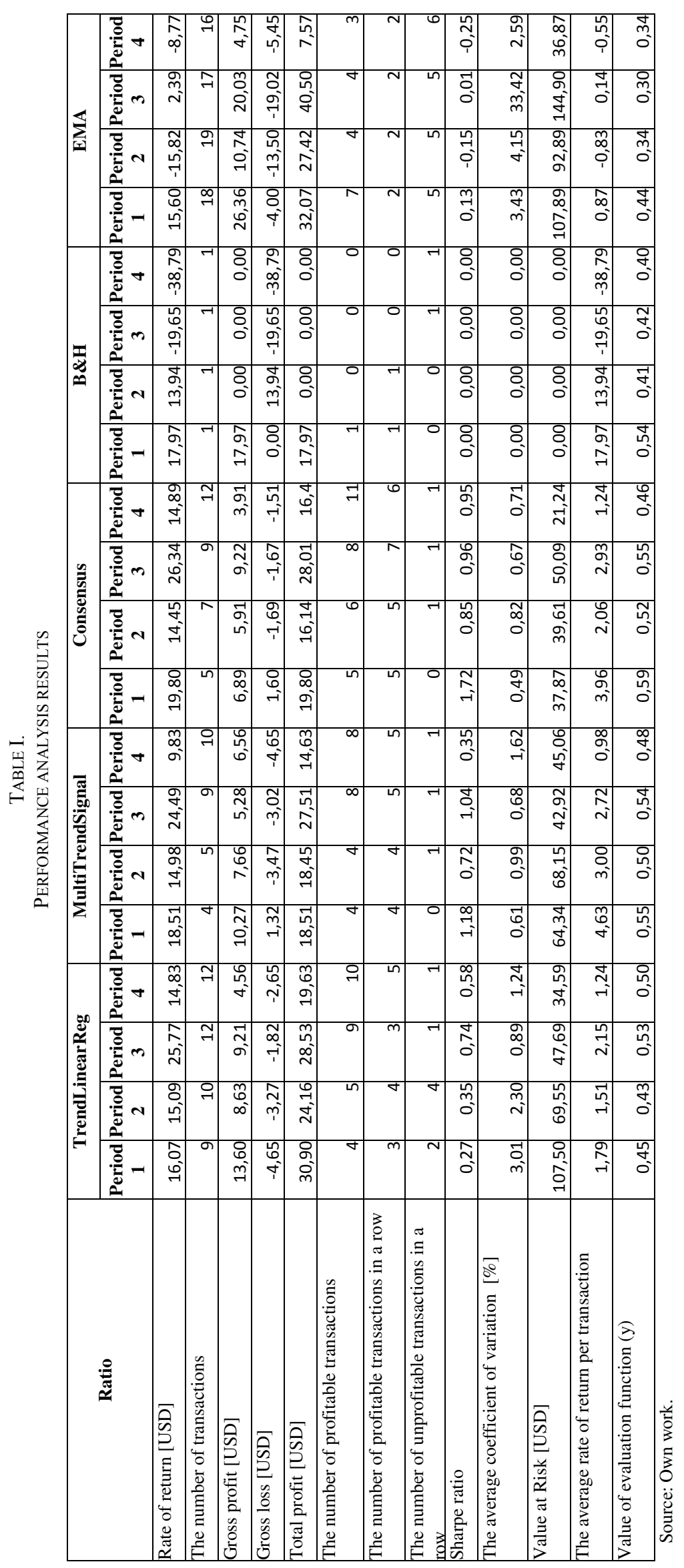


coordinates are used, which is a common way of visualizing and analyzing multivariate data. An agent in n-dimensional space is represented as a polyline with vertices on the parallel axes; the position of the vertex on the ith ratio corresponds to the ith coordinate of the agent.

It may be noticed that the values of efficiency ratios of particular agents differ in each period and get the values (after normalization) in the range from 0 to 1 . The values of such ratios as $\mathrm{x}_{2}, \mathrm{x}_{3}$ and $\mathrm{x}_{11}$ are approximate in case of all agents and the values of ratios $\mathrm{x}_{5}, \mathrm{x}_{6}, \mathrm{x}_{8}$ are characterized by significant distribution in case of particular agents. It may also be noticed that in case of the agents TrendLinearReg, MultitrendSignal and Consensus the values of ratios $\mathrm{x}_{1}, \mathrm{x}_{2}$, $\mathrm{x}_{3}, \mathrm{x}_{9}$ and $\mathrm{x}_{11}$ are similar in each of the periods in question and the values of ratios $\mathrm{x}_{4}, \mathrm{x}_{5}, \mathrm{x}_{6}, \mathrm{x}_{7}, \mathrm{x}_{8}, \mathrm{x}_{10}$ are characterized by much variability in particular periods. A large scope of changes of ratios significantly hinders the analysis by the user and, as a consequence, prevents taking decisions in time close to real time. And the application of the evaluation function allows for immediate appointment of the agent with the best efficiency. It may be noticed that the evaluation function values oscillate in the range from $0.03-0.59$ thus despite large deviations in the values of particular ratios, the agents are evaluated in the range characterized by a smaller value deviation. The results of the experiment performed allow to state that the ranking of agents' evaluation differs in particular periods. In the first period the Consensus agent turned out to be the best agent and the MultiTrendSignal agent was ranked higher and the TrendLinearReg agent was ranked lower than the $\mathrm{B} \& \mathrm{H}$ benchmark evaluation. The EMA benchmark was ranked the lowest in this period. In the second period the MultiTrendSignal and TrendLinearReg agents and the Consensus agent were ranked higher than the EMA and B\&H benchmarks. Considering the third period it may be noticed that the evaluation ranking is similar to the one in the second period. And in the fourth period the MultiTrendSignal was ranked the highest and the TrendLinearReg and Consensus Agents were ranked higher than the B\&H benchmarks. The EMA benchmark was ranked the lowest in this period.

Taking into consideration all the periods in question it may be stated that the Consensus agent was ranked highest most often ( 3 out of 4 periods) although the rate of return of this agent was not always the highest. This evaluation results, however, from the low level of risk connected with investing on the basis of the Consensus agent decision. And, on the other hand, the TrendLinearReg agent was ranked low most often ( 3 out of 4 periods) because at a relatively high risk level it generated little rate of return. Rates of return obtained by the MultiTrendSignal lower than rates of return of the other two agents may result from the fact that this agent is characterized by a low number of transactions because it takes decisions with the use of a few quotation time slots. It may also be noticed that low evaluation of the EMA benchmark in all periods results not from the level of the rate of return but from a high risk level and a large number of loss transactions in a row.

Referring to the evaluation analysis performed in other systems (e.g. in the MetaTrader system) it should be emphasized that it is, in most cases, performed "manually" by the investor. Due to its time consumption, its utility in the systems operating in real time is very limited. Besides, these systems only offer the functions calculating the basic ratios (rate of return, number of transactions, highest profit, highest loss, total profit, number of profitable transactions, number of profitable transactions in a row, number of loss transactions in a row), and in the a-Trader system also additional ratios are calculated, such as the risk measures (Sharp ratio, average value coefficient, risk exposed value), or the average rate of return from transaction.

The evaluation function, elaborated in this article, enables the measurement and the performance evaluation of particular agents taking the buy-sell decision in the system. These operations are made automatically, in time close to real time, by the Supervisor agent which may then suggest the investor taking final decisions on the basis of decisions generated by the agent with the highest level of performance. In addition, enabling the user to change $\mathrm{a}_{\mathrm{i}}$ and $\mathrm{x}_{\mathrm{i}}$ parameters

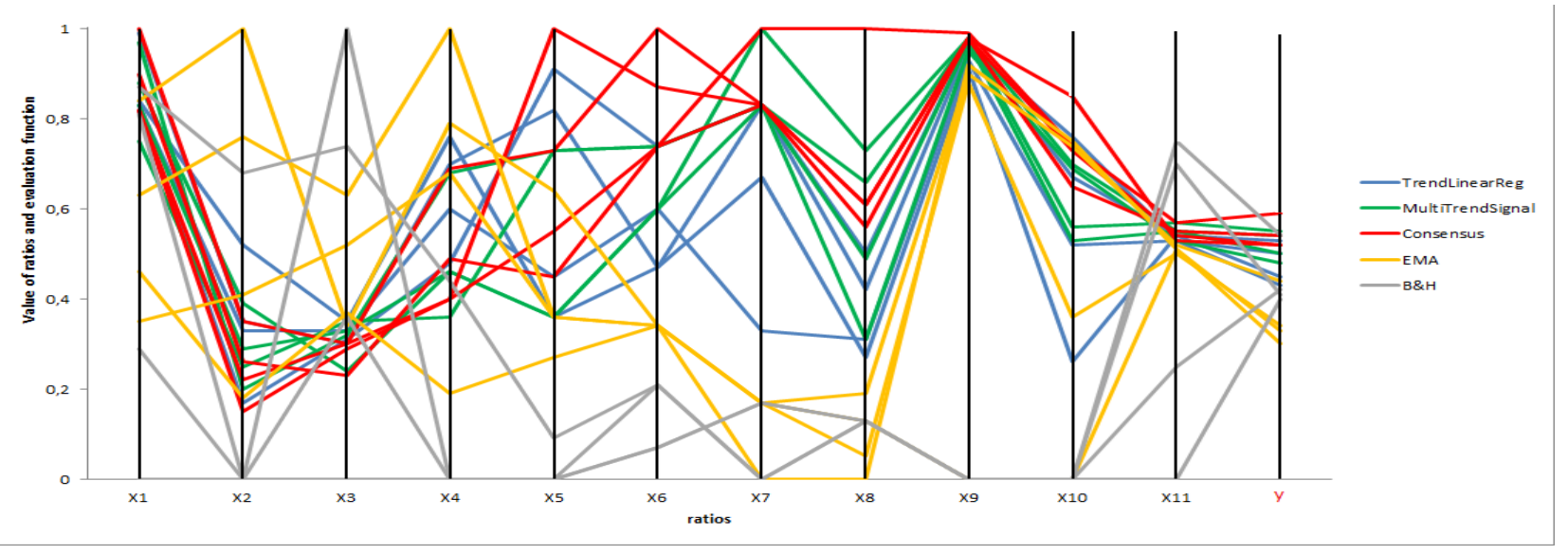

Fig. 5 Values of ratios and evaluation function of particular agents in the periods in question. Source: Own work. 
of the evaluation function allows for considering his/her preference concerning the criterion of importance of particular evaluation ratios. The evaluation value also considers the transaction costs with the assumption that the dependency between the number of transactions and the average rate of return from the transaction is reflected. However this simple principle cannot be adopted because a large number of transactions has impact on the reduction of the agent's efficiency level, especially for the transactions with a high rate of return.

The elaborated evaluation function may be extended with other ratios which do not have directly or (reversely) proportional impact on the function value. For example, this may be the correlation between the rate of return and the ratios defining the risk.

\section{V.CONCLUSION}

The agents in the a-Trader system take independent buysell decisions using various methods for this purpose. The functioning of these agents involves, however, the need to perform constant analysis of their performance, which should be performed by the Supervisor agent. As a consequence, this enables the investor to present decisions generated by the best agents. The analysis results presented in this article allow to draw conclusions that, depending on the current situation on the FOREX market, the level of performance of particular agent changes. There is no agent which definitely dominates over the other ones. And the use of this performance evaluation function allows for automatic setting of the best agent in time close to real time, which has, in turn, a positive influence on investment effectiveness.

Agents based on artificial intelligence methods also function in the a-Trader system. Neural networks recognize the models or sequences of changes of agent signals and on this basis they take a decision. Evolution algorithms are developed, which are able to calculate most effective combinations of agents over a few hundred seconds. Owing to this they adjust to the variable situations dynamically. intelligent methods will be described in the successive articles,

Currently works are being performed on the implementation of the "directional change algorithm" [6], the evolution method of determining $a_{i}$ coefficients into the aTrader system and the implementation of cognitive agents, performing the fundamental analysis and analysing experts' opinions in the scope of forecasts referring to quotations on the FOREX market.

\section{REFERENCES}

[1] M. Aloud, E.P.K. Tsang, R. Olsen, "Modelling the FX Market Traders' Behaviour: An Agent-based Approach", [in] AlexandrovaKabadjova B., S. Martinez-Jaramillo, A. L. Garcia-Almanza \& E. Tsang (eds.), Simulation in Computational Finance and Economics: Tools and Emerging Applications, IGI Global, 2012, pp. 202-228.

[2] R.P. Barbosa, O. Belo, "Multi-Agent Forex Trading System", [in] Agent and Multi-agent Technology for Internet and Enterprise
Systems, Studies in Computational Intelligence Volume 289, 2010, pp. 91-118.

[3] J. Bollinger, "Bollinger on Bollinger Bands", McGraw Hill, 2001.

[4] L. Chan, A. Wk Wong, "Automated Trading with Genetic-Algorithm Neural-Network Risk Cybernet-ics: An Application on FX Markets", Finamatrix, January 2011, pp.1-28.

[5] M. Dempster, C. Jones, "A Real Time Adaptive Trading System using Genetic Programming", Quantitative Finance, 1, 2001, pp. 397-413. DOI: doi:10.1088/1469-7688/1/4/301.

[6] J. B. Glattfelder, A. Dupuis, R. Olsen, "Patterns in High-Frequency FX Data: Discovery of 12 Empirical Scaling Laws", Quantitative Finance, Volume 11 (4), 2011, pp. 599-614,

[7] M. Hernes, Metody consensusu w rozwiązywaniu konfliktów wiedzy $\mathrm{w}$ wieloagentowym systemie wspomagania decyzji, Praca dokt., Uniwersytet Ekonomiczny we Wrocławiu, 2011.

[8] M. Hernes M., N.T. Nguyen, "Deriving Consensus for Hierarchical Incomplete Ordered Partitions and Coverings", Journal of Universal Computer Science 13(2) /2007, pp. 317-328.

[9] K. Jajuga, T. Jajuga, Inwestycje: Instrumenty finansowe, ryzyko finansowe, inżynieria finansowa, PWN, Warszawa 2000.

[10] R. Karjalainen, "Using Genetic Algorithms to Find Technical Trading Rules", Journ. of Financial Econ., 51, 1999, pp. 245-271.

[11] C. D. Kirkpatric, J. Dahlquist, :Technical Analysis: The Complete Resource for Financial Market Technicians”, Financial Times Press, 2006.

[12] J. Korczak, P. Lipinski, „Systemy agentowe we wspomaganiu decyzji na rynku papierów wartościo-wych", [in] Rozwój informatycznych systemów wieloagentowych w środowiskach społecznogospodarczych, ed. S. Stanek et al., Placet, 2008, pp. 289-301.

[13] J. Korczak, M. Bac, K. Drelczuk, A. Fafuła, "A-Trader - Consulting Agent Platform for Stock Ex-change Gamblers", [in] Proc. FedCSIS, Wrocław, 2012, pp.963-968.

[14] J. Korczak, M. Hernes, M. Bac, "Risk avoiding strategy in multiagent trading system", [in] Proceedings of Federated Conference Computer Science and Information Systems (FedCSIS), Kraków, 2013.

[15] B. LeBaron, "Active and Passive Learning in Agent-based Financial Markets", Eastern Economic Journal, vol. 37, 2011, pp. 35-43.

[16] C. Lento, "A Combined Signal Approach to Technical Analysis on the S\&P 500". Journal of Business \& Economics Research 6 (8), 2008, pp. 41-51.

[17] S. Martinez-Jaramillo, E.P.K. Tsang, "An Heterogeneous, Endogenous and Co-evolutionary GP-based Financial Market", IEEE Transactions on Evolutionary Computation, Vol.13, No.1, 2009, pp.33-55.

[18] N. T. Nguyen, "Using Consensus Methodology in Processing Inconsistency of Knowledge", [in] Last M. et al. (Eds): Advances in Web Intelligence and Data Mining, series Studies in Computational Intelligence, Springer-Verlag, 2006, pp. 161-170.

[19] I. Palit, S. Phelps, W. L. Ng, "Can a Zero-Intelligence Plus Model Explain the Stylized Facts of Financial Time Series Data?", [in] Proceedings of the Eleventh International Conference on Autonomous Agents and Multi-Agent Systems (AAMAS) 2012 - Volume 2. Valencia, Spain: International Foundation for Autonomous Agents and Multiagent Systems, pp. 653-660.

[20] J. Sobieska-Karpińska, M. Hernes, "Consensus determining algorithm in multiagent decision support system with taking into consideration improving agent's knowledge", [in] Proceedings of the Federated Conference on Computer Science and Information Systems (FedCSIS), 2012, pp. 1035-1040.

[21] TrendLinearReg, http://forexwikitrading.com/forexindicator/trendlinearreg/ [access: 2014.02.02].

[22] M. Żytniewski, R. Kowal, A. Sołtysik, "The Outcomes of the Research in Areas of Application and Impact of Software Agents Societies to Organizations so far. Examples of Implementation in Polish Companies", [in] Annals of Computer Science and Information Systems, Proceedings of Federated Conference Computer Science and Information Systems (FedCSIS), Kraków, 2013 pp. 1165 - 1168. 